\title{
Carta Editorial
}

\section{Historia Ambiental Aplicada para la Resiliencia Social en Tiempos de Colapso y de Transiciones Socio Ecológicas}

Martha Micheline Cariño-Olvera ${ }^{1}$, Adrián Gustavo Zarrilli ${ }^{2}$, Wilson Picado Umaña ${ }^{3}$, Antonio Ortega Santos ${ }^{4}$, Marina Miraglia ${ }^{5}$, Sandro Dutra e Silva ${ }^{6}$

a edición correspondiente al volumen 11, número 2 (2021) de HALAC: Historia Ambiental Latinoamericana y Caribeña presenta trece artículos, dos reseñas y una entrevista. Ocho de estos artículos son contribuciones al Dossier "Historia

\footnotetext{
${ }^{1}$ Doctora en Historia (École des Hautes Études en Sciences Sociales, Francia), Profesora-Investigadora de la Universidad Autónoma de Baja California Sur, ORCID: https://orcid.org/0000-0003-2627-9508, E-mail: marthamichelinecarino@gmail.com

2 Doctor en Historia (Universidad Nacional de La Plata). Profesor de Historia Argentina en las Universidades Nacionales de Quilmes y La Plata. ORCID: 0000-0002-5830-1303, E-mail: azarrilli@unq.edu.ar

${ }^{3}$ Doctor en Historia (Universidad de Santiago de Compostela, España). Profesor de la Escuela de Historia y coordinador del Observatorio de Historia Agroecológica y Ambiental, de la Universidad Nacional, Costa Rica. ORCID: https://orcid.org/0000-0003-3882-1843. E-mail: wpicado@gmail.com

4 Doctor en Geografía e Historia (Universidad de Granada). Profesor Titular de Historia Contemporánea de la Universidad de Granada e Investigador Responsable del Grupo HUM 952 STAND (South Training Action Network of Decoloniality). ORCID: http://orcid.org/0000-0002-58158500, E-mail: aortegas@ugr.es.

${ }^{5}$ Doctora en Filosofía y Letras con Orientación en Geografía (Universidad de Buenos Aires). Profesora Asociada en la Universidad Nacional de General Sarmiento y docente de la Universidad Nacional de Quilmes, Argentina. ORCID: 0000-0002-8494-6850, E-mail: mmiragli@campus.ungs.edu.ar

${ }^{6}$ Doctor en Historia (Universidad de Brasilia).Docente en la Universidad Estadual de Goiás - UEG y en el Centro Universitario de Anápolis UniEVANGÉLICA (Brasil).Becario de Productividad en Investigación del CNPq. ORCID: 0000-0002-0001-5726, E-mail: sandrodutr@hotmail.com
} 
Ambiental aplicada para la resiliencia social en tiempos de colapso y de transiciones socio ecológicas" y cinco son artículos libres.

En tan solo seis décadas, desde 1960 a la fecha, se pasó de la crisis ecológica, a la civilizatoria y, finalmente, al colapso, del cual esta pandemia de COVID 19, así como el rápido avance del cambio climático son una muestra innegable. Es un cambio de época y no una época de cambios, como bien lo ha mencionado Guillermo Castro reiteradas veces. Una de las características más problemáticas de este proceso es la inevitable incertidumbre, así como la imperiosa necesidad de aprender a vivir con ella.

Este número especial estuvo coordinado por los investigadores Micheline Cariño Olvera (Universidad Autónoma de Baja California Sur, México), Adrián Zarrilli (Universidad Nacional de Quilmes, Argentina), Wilson Picado (Universidad Nacional de Costa Rica) y Antonio Ortega (Universidad de Granada, España).

El dossier está formado por ocho artículos, donde el primero, escrito por Manuel González de Molina, Gloria Guzmán Casado, David Soto Fernández, Juan Infante Amate y Guiomar Carranza Gallego, titulado "Una Aproximación Biofísica a la Industrialización de la Agricultura Española desde la Historia Aplicada", da a conocer los resultados de dos investigaciones realizadas sobre la evolución de la agricultura española y el cambio experimentado hacia un modelo de agricultura industrializada. Los hallazgos cuestionan que el proceso de industrialización y la consiguiente aplicación de las tecnologías de la Revolución Verde dieran lugar a un importante incremento de los rendimientos por unidad de superficie de los principales cultivos.

En su trabajo "Historia y Violencia: Asesinatos de Líderes Indígenas Guardianes del Medio Ambiente en América Latina, 2016-2019", Juan Guillermo Mansilla Sepúlveda, Claudia Andrea Huaiquián Billeke, Diana Elvira Soto Arango, Juan Carlos Beltrán Véliz y Sonia Valle de Frutos plantean una reflexión acerca de la sabiduría ancestral indígena de mujeres y hombres con profundo conocimiento del mundo natural que fueron asesinados recientemente en América Latina. Los resultados pueden contribuir a promover el interés por estudiar los liderazgos de los pueblos originarios y, a la vez, interpela a la comunidad académica y política respecto a los diferentes asesinatos de líderes y lideresas indígenas en diversos territorios de 
América del Sur. A la vez, contribuye a la visibilidad, reconocimiento y difusión del patrimonio cultural inmaterial de la diversidad presente en la América Indígena.

El trabajo de Rolando Canizales Vijil, "Berta Cáceres, Protesta Ambiental en Honduras y la Construcción de Resistencias Comunitarias (1876-2016)" examina la protesta ambiental en Honduras desde la Reforma Liberal de 1876 hasta el asesinato de la líder indígena Berta Cáceres en 2016. A esto se añade una revisión de las políticas sobre la propiedad de la tierra del Estado hondureño. Se explican ciclos de revitalización de una protesta ambiental de signo comunitario con la creación del Consejo Cívico de Organizaciones Populares e Indígenas de Honduras (COPINH), fundado por Cáceres y las políticas del sistema liberal concesionario en los últimos años que indican, sobre todo a partir del Golpe de Estado de 2009, un cambio en el ordenamiento político estructural de las modalidades de explotación de la naturaleza.

Por su parte, Georgina Alethia Sánchez-Reyes, Conrado Márquez-Rosano, María del Carmen Legorreta-Díaz, Manuel Roberto Parra-Vázquez e Ingrid Erin Jane Estrada-Lugo presentan "Mirando el Paisaje desde la Historia Socioambiental. Reconfiguraciones Territoriales en las Cañadas de Ocosingo, Chiapas (1995-2018)". En este trabajo pretenden explicar la historia socioambiental de las cañadas de Avellanal y Taniperlas, y las relaciones sociales en las que el cambio en el paisaje se expresa, mediante observación participante, recorridos de campo, entrevistas y reuniones con sujetos clave, revisión documental e imágenes satelitales. El contexto regional en este caso cobra relevancia y su consideración es necesaria en la elaboración de propuestas de desarrollo rural.

Martha Micheline Cariño-Olvera y Diego Ramírez-Meza en su trabajo "Historia Ambiental Colectiva y desde Abajo: Aplicaciones para la Resignificación Territorial en el Golfo de California" presentan al Golfo de California (GdC) como uno de los cinco ecosistemas marinos con mayor productividad y biodiversidad del planeta. En 2005, sus áreas naturales protegidas fueron inscritas en la lista de Patrimonio de la Humanidad de la UNESCO y alberga numerosos Sitios Ramsar. Sin embargo, contrasta la realidad histórica de una región en la que desde el siglo XVI prevalece la asimetría del poder económico y político. Se han explotado sus recursos y habitantes con severos costos socioecológicos. La propuesta metodológica que presentan los autores 
tiene la intención de hacer de la historia ambiental una herramienta para la resignificación territorial y la lucha por la autonomía de las comunidades del GdC.

En el sexto artículo de este dossier, Wilson Picado Umaña y Carlos Cruz Chaves escriben "Incendiarismo y Pirocultura en el Bosque Tropical Seco de Costa Rica. La Historia del Fuego como Historia Aplicada", donde analizan la evolución de los incendios forestales en Costa Rica desde la perspectiva de la Historia Aplicada. Su objetivo es relacionar el desarrollo de los incendios forestales con los cambios ocurridos en la estructura agraria del país en los últimos años. El estudio se fundamenta en la revisión de fuentes primarias y secundarias, así como de mapas y estadísticas públicas.

El trabajo de Antonio Ortega Santos se titula "Historia Ambiental Aplicada. Herramientas Digitales para el Estudio de la Injusticia Ambiental. Estudios de Caso y Agenda de Humanidades Digitales Ambientales". Aquí abordan elementos centrales a la hora del diseño de nuevas prácticas de investigación, tanto en el ámbito epistemológico como metodológico de la Historia Ambiental. De forma sistemática se plantean dos elementos reflexivos y tres retos de investigación. Como corolario se apunta hacia una propuesta de agenda de futuro para el diseño de proyectos de Historia Pública, enfocados desde la Ciencia Ciudadana que puede ser de utilidad en su aplicación al campo de los estudios socioambientales.

Para finalizar el Dossier, Luis Sánchez Vázquez y Helios Escalante Moreno, en "Más allá de las Centrales Nucleares: Cartografía de Conflictos y Resistencias Frente a la Presencia Radiactiva en Andalucía (España)" presentan una reflexión sobre algunos de los conflictos socioambientales más relevantes relacionados con distintas fases del ciclo nuclear y sus aplicaciones militares en suelo español. En este artículo presentan cuatro conflictos diferentes, describiendo sus principales afectaciones sociales y ambientales y los movimientos de resistencia que surgen para reclamar reconocimiento y reparación. Para ello, los autores emplearon un relato historiográfico que combina los enfoques de la Ecología Política y la Historia Ambiental.

En cuanto a los artículos libres, Valdir Fernandes, Cleverson Vitorio Andreoli, Gilda Collet Bruna e Arlindo Philippi Jr "History and Evolution of the Environmental 
Management System in Brazil" plantean que el establecimiento de sistemas de gestión ambiental en la mayoría de los países fue un reflejo directo de una agenda internacional lanzada durante la Conferencia de Estocolmo, en 1972, que continuó con varias otras conferencias y protocolos, como Río-92, Río + 20, Protocolo de Kioto, París Acuerdo, entre otros, que ha consolidado una agenda ambiental internacional durante los últimos 50 años. En Brasil, este proceso generó reflejos directos con la construcción de un importante marco legal y aparato institucional.

En el artículo "Por uma História Ambiental da Formação Social do Extremo Sul da Bahia (1945-1972)", Marcio Soares Santos y Herbert Toledo Martins abogan por la investigación ambiental histórica de una formación social. Afirman que no hay producción humana sin consecuencias para el medio natural y producido, así como para el propio ser social; así como la organización de la vida social encuentra obstáculos en las condiciones ambientales existentes. Sin embargo, no basta con integrar la naturaleza en la sociedad. Presentan el caso de la explotación depredadora y sistemática de la Mata Atlántica en el extremo sur de Bahía que se llevó a cabo por la acción de madereros y ganaderos, entre la década de 1940 y principios de la de 1970. Buscan en este trabajo señalar algunas cuestiones teóricas en el estudio de una formación social particular, el extremo sur de Bahía, a través de la historia ambiental.

André Felipe Silva y Gabriel Lopes presentan "Entre Horizontes e Sedimentos: o Impacto do Antropoceno na História a partir de Chakrabarty e seus Interlocutores" donde presentan cómo la creciente discusión del término Antropoceno se ha expandido en el campo de las humanidades en los últimos 10 años a partir de las reflexiones pioneras de Chakrabarty. Así muestran cómo los debates que giran en torno a las propuestas de Chakrabarty hacen replantearse varios supuestos fundamentales de la historia, y se centran en la controversia sobre el papel del capitalismo y las escisiones intrahumanas en el nuevo régimen geológico, la distinción entre dimensiones globales y planetarias y el desafío que plantean las inconsistencias entre tiempo histórico y geológico, así como las propuestas analíticas que buscaban responder a las preguntas planteadas por Chakrabarty.

"El Cambio de la Actividad Agrícola en los Llanos de Apan: el Caso del Cultivo del Maguey en el Siglo XX" escrito por Rodolfo Ramírez Rodríguez muestra como los 
espacios geográficos dedicados a un solo cultivo extenso en México, han podido cambiar en menos de una generación humana, esto debido a una serie de alteraciones tanto en la preferencia de cultivos, como en el cambio de uso de suelo y de la propiedad. A pesar de hallarse en la memoria mexicana la imagen de una región donde se privilegiaba el cultivo del maguey de aguamiel, base para la elaboración de la bebida del pulque, esta fue modificada por completo por el incentivo comercial de semillas anuales, siendo favorecida la cebada, precisa para la industria de la cerveza. Por otra parte, la extensión necesaria de los campos de cultivo de maguey, que requería tanto un escalonamiento de plantas, según su edad, como una continua supervisión para ser aprovechadas después de una década, debido a su lento desarrollo, chocó con los intereses inmediatos de los beneficiados por un reparto agrario de las tierras otrora de las haciendas pulqueras.

Vanessa Schweitzer dos Santos, Gabriel Grabowski y Jairo Lizandro Schmitt presentan el artículo titulado "Análise da Realização de Educação Ambiental em uma Rede Pública de Ensino: Contribuições de um Modelo Permanente e Coletivo". Plantean que la Educación Ambiental (EA) debe ser permanente e interdisciplinar en los planes de estudio escolares, promoviendo buenas prácticas ambientales a los estudiantes. En este trabajo analizaron la EA realizada en la Red Municipal de Docencia de Novo Hamburgo / Brasil, con cuestionarios y entrevistas aplicados a los docentes. Las prácticas educativas más frecuentes estaban asociadas con los residuos, el cultivo de huertas y la interacción entre los estudiantes y la naturaleza. Estas acciones fueron bien aceptadas por la comunidad y resultaron en mejoras en el entorno escolar. La organización de estas actividades en forma de Red Docente, a través del Coletivo Educador Ambiental, puede ser un modelo para el desarrollo de la EA en las redes de educación pública.

En cuanto a las reseñas, Gabriel Pereira de Oliveira con el título "Para Trazer o Emaranhado do Mundo ao Ensino de História" realizo la revisión del libro de WAKILD, Emily; BERRY, Michelle K. A Primer for Teaching Environmental History: Ten Design Principles. Durham \& London: Duke University Press, 2018". Por otro lado, André Vasques Vital titula su revisión como "Propondo uma História Ambiental Latinoamericana" y la realiza sobre el libro de John Soluri, Claudia Leal and José 
Augusto Pádua, eds., A Living Past: Environmental Histories of Modern Latin America (New York: Berghahn Books, 2018).

Se cierra esta edición con la Entrevista realizada por Claudia Maria Leal León a Regina Horta Duarte titulada "Encuentros con Circos, Científicos y Animales: el Fecundo Recorrido de Regina Horta Duarte". Cabe mencionar que Regina es una de las fundadoras de la Sociedad Latinoamericana y Caribeña de Historia Ambiental (SOLCHA), profesora de la Universidad Federal de Minas Gerais, en Brasil y editorajefa fundadora de la revista HALAC.

El equipo editorial de HALAC desea que estas contribuciones reciban amplia visibilidad y contribuyan al desarrollo de los debates en todos los espacios académicos, científicos, políticos y sociales interesados en estos temas. 